\title{
Anaerobic adaptation of Mycobacterium avium subspecies paratuberculosis in vitro: similarities to M. tuberculosis and differential susceptibility to antibiotics
}

\author{
Nicole Parrish ${ }^{1^{*}} \mathbb{D}$, Aravinda Vadlamudi ${ }^{1}$ and Neil Goldberg ${ }^{2}$
}

\begin{abstract}
Background: Mycobacterium avium subspecies paratuberculosis (MAP) is the causative agent of Johne's disease in ruminants and is associated with Crohn's disease (CD) in humans, although the latter remains controversial. In this study, we investigated the ability of MAP to adapt to anaerobic growth using the "Wayne" model of non-replicating persistence (NRP) developed for M. tuberculosis.

Results: All strains adapted to anaerobiosis over time in a manner similar to that seen with MTB. Susceptibility to 12 antibiotics varied widely between strains under aerobic conditions. Under anaerobic conditions, no drugs caused significant growth inhibition ( $>0.5 \mathrm{log}$ ) except metronidazole, resulting in an average decrease of $\sim 2$ logs.

Conclusions: These results demonstrate that MAP is capable of adaptation to NRP similar to that observed for MTB with differential susceptibility to antibiotics under aerobic versus anaerobic conditions. Such findings have significant implications for our understanding of the pathogenesis of MAP in vivo and the treatment of CD should this organism be established as the causative agent.
\end{abstract}

\section{Background}

Crohn's disease $(\mathrm{CD})$ is an incurable, chronic inflammatory disorder of the gastrointestinal tract [1]. Although the etiology of $\mathrm{CD}$ is unknown, the clinical findings in humans resemble those of Johne's disease (JD) in cattle, caused by Mycobacterium avium subspecies paratuberculosis (MAP) [1]. In cattle, MAP was established as the etiologic agent of JD by successful demonstration of Koch's postulates [2]. In humans, MAP as the causative agent of $C D$ has been met with both support and skepticism despite the similarities to JD [3]. Supporting evidence for MAP in the etiology of CD may be found in multiple studies in which the organism has either been cultured from intestinal tissues, breast milk, and the blood of CD patients or MAP DNA/RNA has been

\footnotetext{
*Correspondence: nparrish@jhmi.edu

${ }^{1}$ The Johns Hopkins Medical Institutions, 600 North Wolfe Street, Meyer B1-193, Baltimore, Maryland, USA

Full list of author information is available at the end of the article
}

detected in patient samples versus healthy controls [410]. Exposure to MAP may be more widespread than is recognized since viable organism has been found in potable water, commercial milk, and other dairy products, including those having undergone pasteurization sufficient to kill common contaminating organisms [4, 8, 11-13]. MAP can also persist in the environment for long periods of time in the absence of a host as evidenced by pastures which remain infective for months after removal of all infected animals [14, 15]. Unfortunately, the time required for clearance of MAP in the environment is largely unknown.

Skepticism of MAP as the causative agent of CD stems from several lines of evidence which support a strong role for immune dysregulation and highlight failure to achieve a cure with antimicrobial therapy $[1,16]$. Antimicrobial regimens for treatment of $C D$ have included antibiotics such as rifaximin (RFX), ciprofloxacin (CIP), and metronidazole (MET) given separately or in combination 
for a period of one to several months [18-22]. Other trials have used clarithromycin, clofazimine or rifabutin [23]. Efficacy is variable and relapse a common occurrence once therapy is stopped. Questions surrounding the cause of this relapse remain unanswered. Some investigators have suggested the possibility that MAP may be capable of entering a dormant or non-cultivable state in which reversion to vegetative growth is possible when more favorable environmental conditions are present [14]. Dormancy (also known as 'latency') is well documented with respect to other mycobacterial species such as M. tuberculosis and M. bovis BCG (BCG) [24-26]. These related species possess the ability to transition to a non-replicating 'latent' or persistent state in which metabolism is reduced to an extremely low basal level as part of an adaptive response to anaerobiosis $[25,26]$. These 'latently adapted' organisms can persist for years or decades until reactivation occurs due to a variety of factors including waning of immunosurveillance. Recently, some investigators noted that although most humans appear to be susceptible to MAP infection, few develop clinical signs and symptoms immediately following exposure. The authors postulate that in these individuals a 'latent' rather than an 'active' infection is established; an infection which is controlled rather than eliminated by the immune response [27]. Transition of MAP from a vegetative to a 'latent' state may require environmental signals, such as occurs with $M$. tuberculosis in response to decreasing oxygen concentrations. Once adapted to the 'latent' or non-replicating persistent state, MAP may exhibit differential susceptibility to various antibiotics as has been documented with $M$. tuberculosis. In culture, latently adapted $M$. tuberculosis is not susceptible to the majority of commonly used first-line antimycobacterial drugs; suggesting that this population of bacilli cannot be eliminated by conventional antimicrobial therapy which relies on actively growing bacilli to be effective. Only metronidazole (MET), active in anaerobic but not aerobic conditions, significantly inhibits this population of $M$. tuberculosis in vitro [25, 28]. If MAP were capable of anaerobic adaptation to a non-replicating persistent state as seen in M. tuberculosis and BCG, then most antimycobacterial drug regimens would be insufficient to eradicate this population of organisms [24-26, 28]. Interestingly, metronidazole has shown some efficacy in the treatment of Crohn's disease [17-19].

The goal of this study was aimed at answering two specific questions related to the biology of MAP: (1) this fastidious species is known to grow under aerobic conditions so long as culture medium is supplemented with Mycobactin J; however, can MAP adapt to a 'latent' or non-replicating, persistent state in vitro as is the case with M. tuberculosis and BCG and, (2) if so, what is the susceptibility (in vitro) of MAP to antimycobacterial drugs under aerobic versus anaerobic conditions?

\section{Methods \\ Mycobacterial strains and maintenance conditions}

Five strains of MAP were used in this study including one control strain (19,698, type strain, American Type Culture Collection, ATCC, Rockville, MD), three bovine derived strains designated $\mathrm{B} 1$ through $\mathrm{B} 3$, and one human associated strain (Ben, ATCC 43544). M. bovis BCG (Pasteur, ATCC 35734) was used as a control for all assays as this organism has been well characterized in the Wayne model of non-replicating persistence. All strains were maintained on Herrold's egg yolk agar slants containing $2.0 \mu \mathrm{g} / \mathrm{ml}$ Mycobactin J (BectonDickinson, Sparks, Maryland) at $37^{\circ} \mathrm{C}$ in an atmosphere of $5 \% \mathrm{CO}_{2}$.

\section{Aerobic susceptibility testing}

Aerobic susceptibility testing was conducted using broth microdilution in a 96-well plate format. Briefly, a suspension of each strain to be tested was prepared in Middlebrook 7H9 (M7H9) broth (Difco, Detroit, Michigan) supplemented with $2.0 \mu \mathrm{g} / \mathrm{ml}$ Mycobactin J (Allied Monitor, Fayette, Missouri) and diluted to a final density of $\sim 10^{5} \mathrm{CFU} / \mathrm{ml}$. Plates were inoculated with $100 \mu \mathrm{l}$ of the adjusted suspension and the supplied cover put in place, with subsequent incubations for up to 14 days at $37^{\circ} \mathrm{C}$. All plates were read manually using a mirror box and ambient light. The minimum inhibitory $\left(\mathrm{MIC}_{99}\right)$ concentration was determined by comparing growth in the control wells to growth in antibiotic containing wells; the lowest concentration of each drug tested resulting in $99 \%$ inhibition versus the untreated controls was interpreted as the MIC. Antimicrobial agents and their concentrations tested included AMI (1-64 $\mu \mathrm{g} / \mathrm{ml})$, GEN $(0.5-16 \mu \mathrm{g} / \mathrm{ml})$, CLR $(0.125-16 \mu \mathrm{g} / \mathrm{ml}), \mathrm{EMB}(0.5-32 \mu \mathrm{g} /$ $\mathrm{ml})$, MES $(1.5-25 \mu \mathrm{g} / \mathrm{ml})$, SAL $(1.5-25 \mu \mathrm{g} / \mathrm{ml})$, RIF $(0.12-$ $16 \mu \mathrm{g} / \mathrm{ml})$, RFX $(0.12-16 \mu \mathrm{g} / \mathrm{ml})$, CIP $(0.12-4 \mu \mathrm{g} / \mathrm{ml})$, and MET $(12.5 \mu \mathrm{g} / \mathrm{ml})$. All antimicrobial agents with one exception were obtained from Sigma-Aldrich, St. Louis, Missouri. RFX was supplied by Salix Pharmaceuticals, Raleigh, North Carolina. All assays were performed in triplicate and purity plates were done for each susceptibility test.

\section{Anaerobic studies}

We used the in vitro Wayne model of NRP to determine the ability of MAP to survive under anaerobic conditions $[25,26]$. Briefly, MAP cultures were grown in Dubos Tween-albumin broth supplemented with Mycobactin 
J $(2.0 \mu \mathrm{g} / \mathrm{ml})$. Oxygen was gradually depleted from aerobic, exponentially growing cultures $\left(\sim 10^{6} \mathrm{CFU} / \mathrm{ml}\right)$ by aeration at $250 \mathrm{rpm}$ 's for a period of approximately 10-12 days using Hungate-type anaerobic culture tubes. Optical density and colony forming units were determined to monitor the progression of the cultures from aerobic growth through NRP stages 1 and 2. Methylene blue was used to detect the presence of oxygen in the culture. Complete decolorization was used as an indicator for anaerobiosis. Once anaerobiosis had been established, varying concentrations of each antibiotic were added to respective test cultures at concentrations equivalent to as well as several-fold above the aerobic MIC: AMI (up to $16 \mu \mathrm{g} / \mathrm{ml}$ ), GEN (up to $16 \mu \mathrm{g} / \mathrm{ml}$ ), CLR (up to $8 \mu \mathrm{g} / \mathrm{ml}$ ), EMB (up to $32 \mu \mathrm{g} / \mathrm{ml}$ ), RIF (up to $8 \mu \mathrm{g} / \mathrm{ml}$ ), RFX (up to $8 \mu \mathrm{g} / \mathrm{ml}$ ), and CIP (up to $8 \mu \mathrm{g} / \mathrm{ml}$ ). This was followed by further incubation for an additional $48 \mathrm{~h}$. MES and SAL were not tested in the anaerobic model as they failed to inhibit any growth in the aerobic assay. BCG was tested in parallel as a control to demonstrate that the anaerobic model was performing as expected using isoniazid (INH: $0.1-0.4 \mu \mathrm{g} / \mathrm{ml})$ and RIF $(0.06-0.1 \mu \mathrm{g} / \mathrm{ml})$ since neither drug is effective in killing BCG in the anaerobic model [26]. MET $(12.5 \mu \mathrm{g} / \mathrm{ml})$, which was tested at a single concentration, was used as a positive control since it is only active under anaerobic conditions [25, 26]. For each culture, serial dilutions were made and plated to M7H10 agar. Following $\sim 15$ days of incubation, the $\mathrm{CFU} / \mathrm{ml}$ were determined for each culture condition and compared to the untreated and positive and negative controls. All assays were performed in triplicate.

\section{Results}

Aerobic growth and antibiotic susceptibilities

Aerobic susceptibilities varied widely between strains for most of the antibiotics tested (Table 1). Minimum inhibitory concentrations (MIC's, 99\% inhibition) were most consistent between strains with ciprofloxacin (CIP, range $1-2 \mu \mathrm{g} / \mathrm{ml})$. However, greater heterogeneity in MIC's was noted between MAP strains with the remaining active drugs: rifaximin (RFX, 0.25-1), rifampin (RIF, 0.25-4), amikacin (AMI, 2-8), clarithromycin (CLR, 0.125-2), ethambutol (EMB, 2-16), and gentamicin (GEN, 1-4). No inhibition was seen in any MAP strains exposed to metronidazole (MET), mesalamine (MES), or salicylin (SAL) under aerobic conditions at the highest concentration tested for each drug $(12.5,25$ and $25 \mu \mathrm{g} / \mathrm{ml}$, respectively).

\section{Anaerobic adaptation and antibiotic susceptibilities}

All strains demonstrated adaptation to anaerobiosis similar to that of $M$. tuberculosis and BCG with growth ranging from $\sim 10^{7}$ to $10^{8} \mathrm{CFU} / \mathrm{ml}$ after $\sim 10-14$ days incubation from a starting inoculum of $\sim 10^{6} \mathrm{CFU} / \mathrm{ml}$. As shown in Fig. 1, optical density (OD) and colony forming units (CFU's) increased in parallel until day 10. At this time, CFU's began to level off and the methylene blue began to fade (days 12 through 16). This stage was followed by a slight increase in OD's without concomitant increase in CFU's/ml. For all strains, by days 18-19, the methylene blue had completely faded indicating conversion to anaerobiosis. Anaerobic susceptibilities indicated more homogeneous results than those observed with

Table 1 Minimum inhibitory concentrations to various antibiotics for MAP strains used in this study under aerobic versus anaerobic conditions

\begin{tabular}{|c|c|c|c|c|c|c|c|c|c|c|c|}
\hline \multirow[t]{2}{*}{ MAP strain } & \multirow[t]{2}{*}{ Condition } & \multicolumn{10}{|c|}{ Test ranges and MICs $(\mu \mathrm{g} / \mathrm{ml})$} \\
\hline & & RIF & RFX & AMI & CIP & CLR & EMB & GEN & MET & MES & SAL \\
\hline \multirow[t]{2}{*}{ ATCC 19698} & Aerobic & 2 & 0.5 & 2 & 2 & 0.5 & 4 & 1 & $>12.5$ & $>25$ & $>25$ \\
\hline & Anaerobic & $>8$ & $>8$ & $>16$ & $>8$ & $>8$ & $>16$ & $>16$ & 12.5 & NT & NT \\
\hline \multirow[t]{2}{*}{ B-1 } & Aerobic & 1 & 1 & 4 & 2 & 1 & 8 & 2 & $>12.5$ & $>25$ & $>25$ \\
\hline & Anaerobic & $>8$ & $>8$ & $>16$ & $>8$ & $>8$ & $>16$ & $>16$ & 12.5 & NT & NT \\
\hline \multirow[t]{2}{*}{ B-2 } & Aerobic & 0.25 & 0.25 & 2 & 1 & 0.125 & 2 & 2 & $>12.5$ & $>25$ & $>25$ \\
\hline & Anaerobic & $>8$ & $>8$ & $>16$ & $>8$ & $>8$ & $>16$ & $>16$ & 12.5 & NT & NT \\
\hline \multirow[t]{2}{*}{ B-3 } & Aerobic & 2 & 0.5 & 4 & 2 & 2 & 16 & 2 & $>12.5$ & $>25$ & $>25$ \\
\hline & Anaerobic & $>8$ & $>8$ & $>16$ & $>8$ & $>8$ & $>16$ & $>16$ & 12.5 & NT & NT \\
\hline \multirow[t]{2}{*}{ ATCC 43544} & Aerobic & 4 & 1 & 8 & 1 & 1 & 8 & 4 & $>12.5$ & $>25$ & $>25$ \\
\hline & Anaerobic & $>8$ & $>8$ & $>16$ & $>8$ & $>8$ & $>16$ & $>16$ & 12.5 & NT & NT \\
\hline
\end{tabular}

The concentrations shown are the highest tested for each antibiotic in the anaerobic model

M. bovis BCG ATCC 35734 (Pasteur) was used as a control for the anaerobic model as previously described [45]. Aerobic MICs for BCG were $<0.1$ for isoniazid and $\leq 0.06$ for rifampin. Neither drug significantly inhibited growth of BCG in the anaerobic model at concentrations above (INH $>0.4 ; \mathrm{RIF}>0.1)$ that observed under aerobic conditions

RIF rifampin, RFX rifaximin, AMI amikacin, CIP ciprofloxacin, CLR clarithromycin, EMB ethambutol, GEN gentamicin, MET metronidazole, MES mesalamine, SAL salicilin, $N T$ not tested 


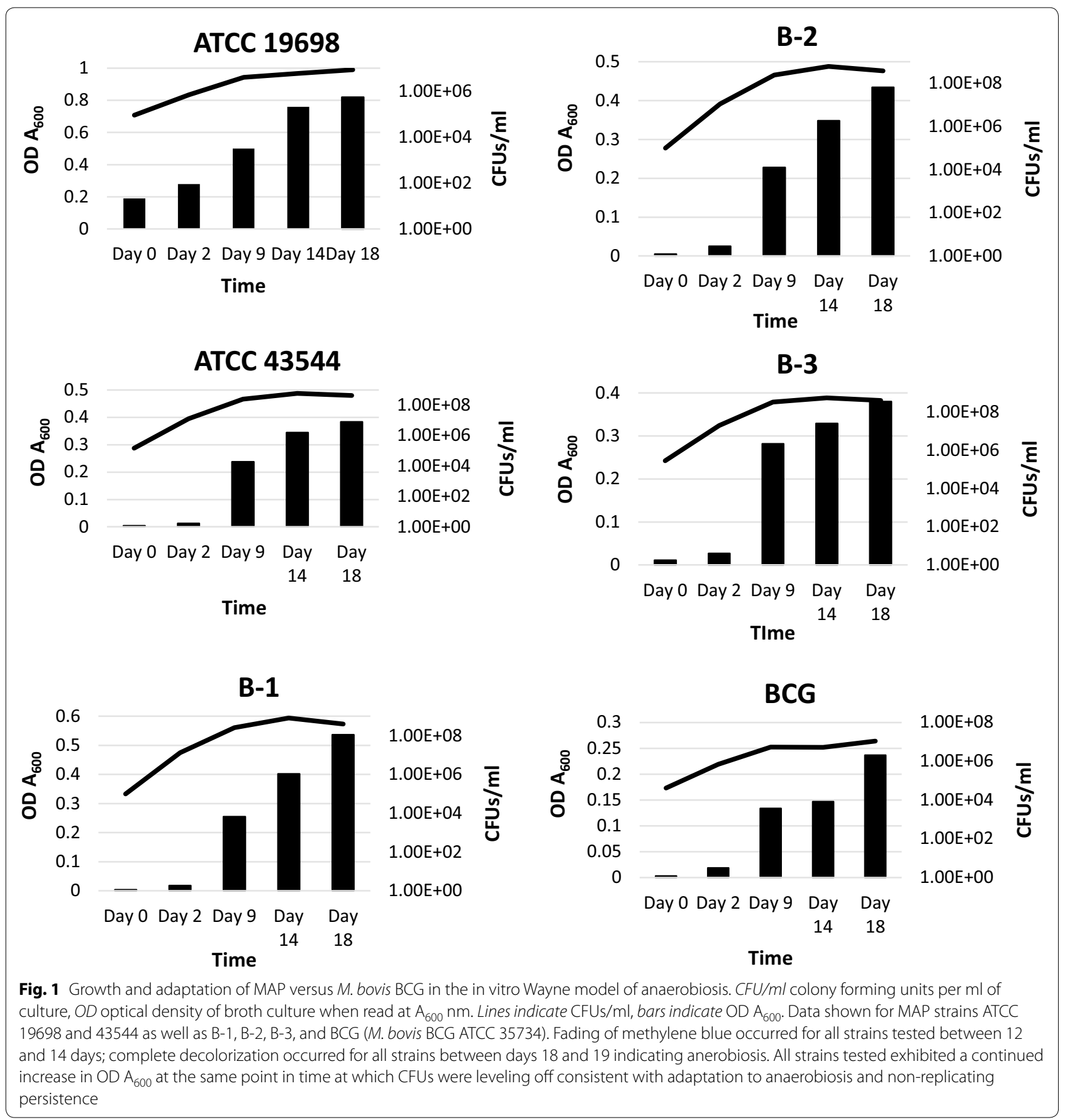

aerobically growing cultures. No drugs tested resulted in $99 \%$ inhibition of growth; thus an $\mathrm{MIC}_{99}$ could not be established under anaerobic conditions (Table 1). Only MET $(12.5 \mu \mathrm{g} / \mathrm{ml})$ showed appreciable activity with a 2- $\log _{10}$ drop in CFU's/ml following $48 \mathrm{~h}$ exposure. Of the other drugs tested, only RIF, and RFX showed minimal activity with $0.5 \log _{10}$ inhibition of growth. However, inhibition with RIF and RFX required the use of concentrations 1- to 8-fold above the MIC for each drug compared with aerobically growing cultures. No activity was observed with the remaining drugs: AMI, CIP, CLR, EMB, and GEN with $<0.5 \log$ decrease in CFU $/ \mathrm{ml}$. MES and SAL were not tested in the anaerobic model as they were not active against aerobically growing strains. 


\section{Discussion}

The results from this study demonstrate that MAP is capable of both aerobic growth and adaptation in anaerobiosis when using the 'Wayne' in vitro model developed for M. tuberculosis. This particular model illustrates the progression of $M$. tuberculosis through two stages of nonreplicating persistence (NRP) in response to the gradual withdrawal of oxygen from a culture over a period of days to weeks. Gradual withdrawal of oxygen permits time for genetic and phenotypic adaptation to anoxic conditions via progression through two stages of NRP [25]. NRP-1 is a microaerophilic state in which culture OD's increase, although at a slower rate than during vegetative growth, without a concomitant increase in CFU's/ $\mathrm{ml}$. NRP-2 is characterized by adaptation to complete anaerobiosis with no further increase in OD [25]. In the current study, not only did MAP demonstrate clear progression though both NRP-1 and NRP-2, but also in much the same manner as that observed with $M$. tuberculosis. It has been suggested that the ability of $M$. tuberculosis to adapt to NRP-1 and NRP-2 is responsible for the survival of the organism over long periods of time in the human host. Reversion to a vegetative state occurs when conditions are more favorable leading to reactivation of 'latent' disease. In this way, M. tuberculosis is able to persist in tissues for months to years without replicating or causing active disease while remaining immunologically antigenic. Recently, Janagama et al. examined the transcriptome of MAP in vivo and found that transcripts pertaining to latency were upregulated in intestinal tissues indicating that regulation of these specific pathways appear to be related to tissue and cell type [29]. They showed that MAP obtained from tissues exhibited significant "shutdown" of major metabolic pathways suggesting that MAP survival was directly correlated with the ability to evade the immune response by entering a persistent state and surviving within macrophages [29, 30]. Investigators have also noted that most humans appear to be susceptible to MAP infection; however, few develop clinical signs and symptoms immediately following exposure [16, 31-34]. Although the reasons for this delay have not been elucidated, one possibility is that any MAP present have entered a state of NRP and much like $M$. tuberculosis, establish a 'latent' infection which the immune response is unable to eliminate. Waning of immune surveillance would necessarily lead to a reversal of NRP and potential development of active disease. Further complicating the picture in $C D$ is the presence of polymorphisms in the NOD2/CARD15 gene which have been identified in those with the disease versus paired controls [35]. Not only may NOD2/CARD15 play a role in the defense against pathogenic bacteria, but more importantly provide for regulation of mediators of intestinal inflammation. The presence of anaerobically adapted MAP in intestinal tissues may lead to persistent antigenic stimulation in the absence of cultivable bacilli not unlike that observed in those with latent $M$. tuberculosis infection. Recently, a large meta-analysis was performed which investigated genome-wide association scans of over 75,000 cases/controls of CD and ulcerative colitis (UC) patients. This study found a strong association between $\mathrm{CD} / \mathrm{UC}$ with genes involved in primary immunodeficiencies. Interestingly, a subset of the genes involved in primary immunodeficiencies was also associated with increased susceptibility to mycobacterial infection suggesting that those with $\mathrm{CD} / \mathrm{UC}$ may be more susceptible to not only MAP but also M. tuberculosis as well [36].

Further similarities between MAP and M. tuberculosis were demonstrated in the present study by the differential susceptibility to antibiotics observed under aerobic versus anaerobic conditions. When completely adapted to anaerobiosis, $M$. tuberculosis is "impervious" to the activity of most aerobically active drugs as seen with MAP strains tested in this study [25, 28]. Only MET exhibited any activity against anaerobically adapted MAP strains, findings consistent with those observed for $M$. tuberculosis in the same model [25]. MET, is a prodrug which requires activation under anaerobic conditions [25]. Thus, the lack of activity observed for MET with aerobically growing MAP strains is consistent with the known mechanism of action of this drug. In addition, the $\log _{10}$ reduction in CFU's/ml observed in MET exposed MAP under anaerobic conditions closely mirrored that seen with BCG, used as a control in this study.

This study does have some limitations. First, MES failed to inhibit any of the MAP strains tested in this study when grown under aerobic conditions; thus this drug was not tested in the anaerobic model. However, previous investigators reproducibly demonstrated weak bacteriostatic activity of MES against aerobically adapted MAP using the sensitive BACTEC $460^{\circledR}$ radiometric system (Becton-Dickinson, Sparks, MD) [37, 38]. Interestingly, the same results were not obtained using the BACTEC Mycobacterial Growth Indicator Tube (MGIT-960 ${ }^{\circledR}$, Becton-Dickinson) [37-39]. It is possible that differences in testing methods are responsible for the variability in MAP susceptibility to MES observed between studies. Unfortunately, the most sensitive of the methods used for MAP susceptibility testing, the BACTEC 460 system, is no longer commercially supported nor are consumables available, which in particular negatively impacts assessment of bacteriostatic drugs. Secondly, a number of investigators have demonstrated dose-dependent inhibition of MAP in culture by a number of anti-inflammatory drugs including methotrexate and 6-mercaptopurine 
[37-44]. As a result, Greenstein et al. postulated that treatment of patients with inflammatory bowel disease with methotrexate and 6-mercaptopurine may result in inhibition of MAP and secondarily a decrease in proinflammatory cytokines [42]. In the current study, testing of anti-inflammatory drugs was beyond the scope of this project. However, future studies are planned which will determine the activity of anti-inflammatory drugs against MAP under both aerobic and anaerobic conditions.

Although a definitive causal link between CD and MAP has not been established, the findings as presented in this investigation raise some interesting questions. Could the presence of anaerobically adapted MAP in intestinal tissues lead to persistent antigenic stimulation and the chronic inflammation observed in CD? Could the variable efficacy of different antibiotic regimens used in $\mathrm{CD}$ patients be related to the presence of mixed populations of aerobically and anaerobically adapted MAP? Is relapse related to 'reactivation' of anaerobically adapted organisms not eliminated by conventional antibiotic regimens? Could this explain why some studies have shown efficacy in CD patients receiving MET? These and other questions require further investigation including prospective, randomized, clinical trials which utilize antimicrobial combination regimens active against both aerobic and anaerobically adapted MAP.

\section{Conclusions}

This study demonstrates the ability of MAP to adapt to anaerobiosis in the in vitro Wayne model developed for M. tuberculosis. Adaptation of MAP to a non-replicating persistent state demonstrated differential susceptibility to antibiotics under aerobic versus anaerobic conditions. This has implications for treatment to eradicate this organism in vivo as standard antibiotics currently used to target MAP have little to no effect against nonreplicating persistent bacilli. Since mixed populations of MAP are likely to exist simultaneously in vivo, combination therapies containing metronidazole and aerobically active drugs such as clarithromycin and rifaximin should be considered.

\section{Authors' contributions}

NP and AV performed assays. AV, NP and NG performed data analysis. NP and NG wrote the manuscript. I confirm that this is my work and has not been published previously. All authors read and approved the final manuscript.

\section{Author details}

${ }^{1}$ The Johns Hopkins Medical Institutions, 600 North Wolfe Street, Meyer B1-193, Baltimore, Maryland, USA. ${ }^{2}$ Saint Joseph Medical Center, University of Maryland, Towson, Maryland, USA.

\section{Acknowledgements}

This research was supported in part by the St. Joseph Medical Center Foundation, Towson, Maryland. We would also like to thank Miss Erin Weddle for her participation as a volunteer on this project. She contributed a number of hours of her time working on a portion of this project.

\section{Competing interests}

The authors declare that they have no competing interests.

\section{Availability of data and materials}

The datasets used and or analyzed during the current study are available from the corresponding author upon reasonable request.

\section{Publisher's Note}

Springer Nature remains neutral with regard to jurisdictional claims in published maps and institutional affiliations.

Received: 25 January 2017 Accepted: 1 June 2017

Published online: 10 June 2017

\section{References}

1. Greenstein RJ. Is Crohn's disease caused by a mycobacterium? Comparisons with leprosy, tuberculosis, and Johne's disease. Lancet Infect Dis. 2003;3:507-14.

2. Taylor AW. Experimental Johne's disease in cattle. J Comp Pathol. 1953;8(4):355-67.

3. Van Kruiningen HJ. Lack of support for a common etiology in Johne's disease of animals and Crohn's disease in humans. Inflamm Bowel Dis. 1999;5:183-91.

4. Mishina D, Katsel P, Brown ST, Gilberts EC, Greenstein RJ. On the etiology of Crohn's Disease. Proc Natl Acad Sci. 1996;93(18):9816-20.

5. Bull TJ, McMinn EJ, Sidi-Boumedine K, Skull A, Durkin D, Neild P, et al. Detection and verification of Mycobacterium avium subsp. paratuberculosis in fresh ileocolonic mucosal biopsy specimens from individuals with and without Crohn's disease. J Clin Microbiol. 2003:41:2915-23.

6. Naser SA, Ghobrial G, Romero C, Valentine JF. Culture of Mycobacterium avium subspecies paratuberculosis from the blood of patients with Crohn'sdisease. Lancet. 2004;364:1039-44.

7. Sechi LA, Scanu AM, Molicotti P, Cannas S, Mura M, Dettori G, et al. Detection and isolation of Mycobacterium avium subspecies paratuberculosis from intestinal mucosal biopsies of patients with and without Crohn's disease in Sardinia. Am J Gastroenterol. 2005; 100:1529-36

8. Ellingson JL, Anderson AL, Koziczkowski JJ, Radcliff R, Sloan SJ, Allen SE, et al. Detection of viable Mycobacterium avium subsp. paratuberculosis in retail pasteurized whole milk by two culture methods and PCR. J Food Prot. 2005;68:966-72

9. Pickup RW, et al. Mycobacterium avium subsp. paratuberculosis in lake catchments, in river water abstracted for domestic use, and in effluent from domestic sewage treatment works: diverse opportunities for environmental cycling and human exposure. Appl Environ Microbiol. 2006;72:4067-77.

10. Abubakar I, Myhill D, Aliyu SH, Hunter PR. Detection of Mycobacterium avium subspecies paratuberculosis from patients with Crohn's disease using nucleic acid-based techniques: a systematic review and metaanalysis. Inflamm Bowel Dis. 2007;14(3):401-10.

11. Pierce ES. Possible transmission of Mycobacterium avium subspecies paratuberculosis through potable water: lessons from an urban cluster of Crohn's disease. Gut Pathog. 2009;1(1):17.

12. Pickup RW, Rhodes G, Arnott S, Sidi-Boumedine K, Bull TJ, Weightman A, et al. Mycobacterium avium subsp. paratuberculosis in the catchment area and water of the river Taff in south Wales, United Kingdom, and its potential relationship to clustering of Crohn's disease cases in the city of Cardiff. Appl Environ Microbiol. 2005;71:2130-9.

13. Donaghy JA, Johnston J, Rowe MT. Detection of Mycobacterium avium subsp. paratuberculosis in cheese, milk powder and milk using IS900 and f57-based qPCR assays. J Appl Microbiol. 2011;110(2):479-89.

14. Whittington RJ, Marshall DJ, Nicholls PJ, Marsh IB, Redacliff LA. Survival and dormancy of Mycobacterium avium subsp. paratuberculosis in the environment. Appl Environ Microbiol. 2004;70:2989-3004.

15. Whittington RJ, Marsh IB, Redacliff LA. Survival of Mycobacterium avium subsp. paratuberculosis in dam water and sediment. Appl Environ Microbiol. 2005;71:5304-8. 
16. Pierce ES. Where Are All the Mycobacterium avium subspecies paratuberculosis in patients with Crohn's Disease? PLoS Pathog. 2009;5(3):e1000234. doi:10.1371/journal.ppat.1000234.

17. Levine A, Turner D. Combined azithromycin and metronidazole therapy is effective in inducing remission in pediatric Crohn's disease. J Crohns Colitis. 2011;5(3):222-6.

18. Sutherland L, Singleton J, Sessions J, Hanauer S, Krawitt E, Rankin G, et al. Double-blind, placebo controlled trial of metronidazole in Crohn's disease. Gut. 1991;32(9):1071-5.

19. Kale-Pradhan PB, Zhao JJ, Plamer JR, Wilhelm SM. The role of antimicrobials in Crohn's disease. Expert Rev Gastroenterol Hepatol. 2013;7(3):281-8.

20. Blichfeldt P, Blomhoff JP, Myhre E, Gjone E. Metronidazole in Crohn's disease. A double-blind, cross-over clinical trial. Scand I Gastroenterol. 1978;13(1):123-7.

21. Prantera C, Lochs H, Grimaldi M, Danese S, Scribano ML, Gionchetti P. Retic study group: rifaximin-extended intestinal release induces remission in patients with moderately active Crohn's disease. Gastroenterol. 2012;142(3):473-81.

22. Guslandi M. Rifaximin in the treatment of inflammatory bowel disease. World J Gastroenterol. 2011;17(42):4643-6.

23. Warwick S, Pavli P, Crotty B, Florin T, Radford-Smith G, Gibson P, et al. Twoyear combination antibiotic therapy with clarithromycin, rifabutin, and clofazimine for Crohn's disease. Gastroenterology. 2007;132:2313-9.

24. Wayne LG. Dormancy of Mycobacterium tuberculosis and latency of disease. Eur J Clin Microbiol Infect Dis. 1994;13:908-14.

25. Wayne $L G$, Hayes $L G$. An in vitro model for sequential study of shiftdown of Mycobacterium tuberculosis through two stages of non- replicating persistence. Infect immun. 1996;64(6):2062-9.

26. Lim A, Eleuterio M, Hutter B, Murugasu-Oei B, Dick T. Oxygen depletion-induced dormancy in Mycobacterium bovis BCG. J Bacteriol. 1999;181:2252-6.

27. Davis WC, Madsen-Bouterse SA. Crohn's disease and Mycobacterium avium subsp. paratuberculosis, the need for a study is long overdue. Vet Immunol Immunopathol. 2012;145(1-2):1-6.

28. Hu Y, Coates A, Mitchison D. Sterilizing activities of fluoroquinolones against rifampin-tolerant populations of Mycobacterium tuberculosis. Antimicrob Agents Chemother. 2003;47:653-7.

29. Janagama HK, Lamont EA, George S, Bannantine JP, Xu WW, Zj Tu, et al. Primary transcriptomes of Mycobacterium avium subsp. paratuberculosis reveal proprietary pathways in tissue and macrophages. BMC Genom. 2010;11:561-71.

30. Cossu A, Sechi LA, Zanetti S, Rosu V. Gene expression profiling of Mycobacterium avium subsp. paratuberculosis in simulated multi-stress conditions and within THP-1 cells reveals a new kind of interactive intramacrophage behavior. BMC Microbiol. 2012;12:87-103.

31. Gelfand MD, Krone CL. Inflammatory bowel disease in a family. Observations related to pathogenesis. Ann Intern Med. 1970;72:903-7.

32. Van Kruiningen HJ, Freda BJ. A clustering of Crohn's disease in Mankato. Minn Inflamm Bowel Dis. 2001;7:27-33.

33. Allan RN, Pease P, Ibbotson JP. Clustering of Crohn's disease in a Cotswold village. Q J Med. 1986;59:473-8.

34. El-Zaatari FAK, Osato MS, Graham DY. Etiology of Crohn's disease: the role of Mycobacterium avium paratuberculosis. Trends Mol Med. 2001;7(6):247-53.

35. Michail S, Bultron G, Depaolo RW. Genetic variants associated with Crohn's disease. Appl Clin Genet. 2013;6:25-32.

36. Franke A, McGovern D, Barrett J, Wang K, Radford-Smith G, Ahmad T, et al. Genome-wide meta-analysis increases to 71 the number of confirmed Crohn's disease susceptibility loci. Nat Genet. 2010;42:11118-25.

37. Greenstein RJ, Su L, Shahidi A, Brown ST. On the action of 5-amino-salicylic acid and sulfapyridine on M. avium including subspecies paratuberculosis. PLoS ONE. 2007;2(6):e516. doi:10.1371/journal.pone.0000516.

38. Greenstein RJ, Su L, Shahidi A, Brown WD, Clifford A, Brown ST. Unanticipated Mycobacterium tuberculosis complex culture inhibition by immune modulators, immune suppressants, a growth enhancer, and vitamins A and D: clinical implications. Int J Infect Dis. 2014;26(C):37-43.

39. Shin SJ, Collins MT. Thiopurine drugs azathioprine and 6-mercaptopurine inhibit Mycobacterium paratuberculosis growth in vitro. Antimicrob Agents Chemother. 2008;52(2):418-26.

40. Greenstein RJ, Cameron DW, Brown ST. Yet another flawed "placebo controlled" study in Crohn's disease? Foodborne Pathog Dis. 2015;12(9):812.

41. Selby W, Pavli P, Crotty B, Florin T, Radford-Smith G, Gibson P, et al. Twoyear combination antibiotic therapy with clarithromycin, rifabutin, and clofazimine for Crohn's disease. Gastroenterol. 2007;132(7):2313-9.

42. Greenstein RJ, Su L, Haroutunian V, Shahidi A, Brown ST. On the action of methotrexate and 6-mercaptopurine on M. avium subspecies paratuberculosis. PLoS ONE. 2007;2(1):e161.

43. Greenstein RJ, Su L, Juste RA, Brown ST. On the action of cyclosporine A, rapamycin and tacrolimus on M. avium including subspecies paratuberculosis. PLoS ONE. 2008;3(6):e2496.

44. Krishnan MY, Manning EJ, Collins MT. Effects of interactions of antibacterial drugs with each other and with 6-mercaptopurine on in vitro growth of Mycobacterium avium subspecies paratuberculosis. J Antimicrob Chemother. 2009;64(5):1018-23.

45. Parrish NM, Ko CG, Dick JD. Activity of DSA against anaerobically adapted Mycobacterium bovis BCG in vitro. Tuberculosis. 2009;89(4):325-7.

\section{Submit your next manuscript to BioMed Central and we will help you at every step:}

- We accept pre-submission inquiries

- Our selector tool helps you to find the most relevant journal

- We provide round the clock customer support

- Convenient online submission

- Thorough peer review

- Inclusion in PubMed and all major indexing services

- Maximum visibility for your research

Submit your manuscript at www.biomedcentral.com/submit
( ) BioMed Central 